\title{
Tetrahedral Zinc Complexes with Liquid Crystalline and Luminescent Properties: Interplay between Non-conventional Molecular Shapes and Supramolecular Mesomorphic Order.
}

Emma Cavero, ${ }^{\dagger}$ Santiago Uriel, ${ }^{\ddagger}$ Pilar Romero, ${ }^{\dagger}$ José Luis Serrano, ${ }^{\dagger}$ and Raquel Giménez ${ }^{*}{ }^{\dagger}$

Departamento de Química Orgánica y Química Física, Área de Química Orgánica, Facultad de Ciencias-Instituto de Ciencia de Materiales de Aragón, Universidad de Zaragoza-CSIC, 50009 Zaragoza, Spain, and Centro Politécnico Superior-Instituto de Ciencia de Materiales de Aragón, Universidad de Zaragoza-CSIC, 50015 Zaragoza, Spain.

\section{CONTENTS}

- Full synthetic and analytical details for all new compounds.

- Materials and methods

- General procedure for the synthesis of the Bpzm ligands (7-12) and characterization data.

- General procedure for the synthesis of the complexes (13-24) and characterization data.

- 1D-ROESY spectra for 12.

- ${ }^{1} \mathrm{H}$ NMR spectra for the characterization of isomers in $\mathbf{8}$ and $\mathbf{2 0 .}$

- Variable temperature ${ }^{1} \mathrm{H}$ NMR spectra for $\mathbf{1 8 .}$

- ORTEP molecular drawings with atom numbering scheme and representative distances for 18.

- ORTEP molecular drawings with atom numbering scheme and representative distances for 24. 
Materials and Methods. All chemical reagents and solvents were obtained from commercial sources and used without purification. Synthetic procedures and characterization data for the precursor pyrazoles Hpz 1-6 have already been reported (Barberá, J.; Giménez, R.; Serrano, J. L. Chem. Mater. 2000, 12, 481-489 and Barberá, J.; Cativiela, C.; Serrano, J. L.; Zurbano, M. M. Liq. Cryst. 1992, 11, 887-897) or were prepared in a similar way.

NMR spectra $\left({ }^{1} \mathrm{H},{ }^{13} \mathrm{C},{ }^{1} \mathrm{H}^{-13} \mathrm{C}\right.$ HSQC, NOE experiments) were measured on Bruker AVANCE 500 and Bruker AVANCE 400 spectrometers. Chemical shifts are given in ppm relative to TMS and the solvent residual peak was used as the internal standard. Chemical shifts were assigned following a lettering scheme displayed next to the characterization data for all compounds. The conformations of ligands and complexes in solution were determined by NOE experiments in one or two dimensions. Selective ${ }^{1} \mathrm{H}$ Double Pulsed-Field Gradient (DPFG) NOESY/ROESY and 2D phase-sensitive ${ }^{1} \mathrm{H}-{ }^{1} \mathrm{H}$ NOESY experiments were carried on samples in $\mathrm{CDCl}_{3}$ at $25^{\circ} \mathrm{C}$ on a $500 \mathrm{MHz}$ Bruker AVANCE spectrometer equipped with a 5-mm inverse probe. The mixing time (between 0.25 and $1.2 \mathrm{~s}$ ) was optimized by ${ }^{1} \mathrm{H} \mathrm{T}_{1}$ measurements and the relaxation delay was $2 \mathrm{~s}$. For 1D-ROESY experiments the selective 180 pulse was of 40 ms duration with a Gaussian-shaped pulse.

Microanalyses were performed with a Pekin-Elmer 2400 microanalyzer. IR spectra were recorded on a Nicolet Avatar FTIR spectrophotometer using $\mathrm{KBr}$ pellets. Mass spectra were obtained on VG Autospec EBE (FAB+) and MICROFLEX Bruker (MALDI+) spectrometers.

Mesophase analysis was performed using a Linkam THMS600 hot stage and an Olympus polarizing microscope equipped with an Olympus DP12 digital camera. Transition temperatures and enthalpies were obtained by differential scanning calorimetry with a Perkin-Elmer DSC-7 apparatus at heating and cooling rates of $10^{\circ} \mathrm{C} / \mathrm{min}$. The apparatus was previously calibrated with indium $\left(156.6^{\circ} \mathrm{C}, 28.44 \mathrm{~J} / \mathrm{g}\right)$. Powder X-ray diffraction patterns were obtained using a pinhole camera (Anton Paar) operating with a point focused Ni-filtered $\mathrm{Cu}-K \alpha$ beam. The sample was held in Lindemann glass capillaries (0.9 and 1 mm diameter) and heated, when necessary, with a variable-temperature attachment. The diffraction patterns were collected on flat photographic film. 
Optical absorption spectra were recorded with a UV4-200 UV-Vis spectrophotometer from ATI-

Unicam. Fluorescence spectra were recorded with a Perkin-Elmer LS50B system. Thin-film spectra were recorded by front-face detection. Single crystal X-ray diffraction data were measured on an Xcalibur S diffractometer from Oxford Diffraction Ltd., using Mo- $K \alpha$ radiation $(\lambda=0.71073 \AA)$.

General procedure for the synthesis of the Bpzm ligands (7-12): Anhydrous $\mathrm{K}_{2} \mathrm{CO}_{3}(20$ mmol) and $\mathrm{TBAB}(0.7 \mathrm{mmol})$ were added to a solution of the corresponding pyrazole $\mathrm{Hpz}(10 \mathrm{mmol})$ in dibromomethane $(15 \mathrm{ml})$. The resulting mixture was heated under reflux and the reaction was monitored by TLC. After stirring for $48 \mathrm{~h}$ the reaction was allowed to cool, extracted with dichloromethane and the organic layer was dried with magnesium sulfate. The solvent was removed under reduced pressure and the crude product was purified by Cromatotron ${ }^{\circledR}$, using the appropriate eluent.

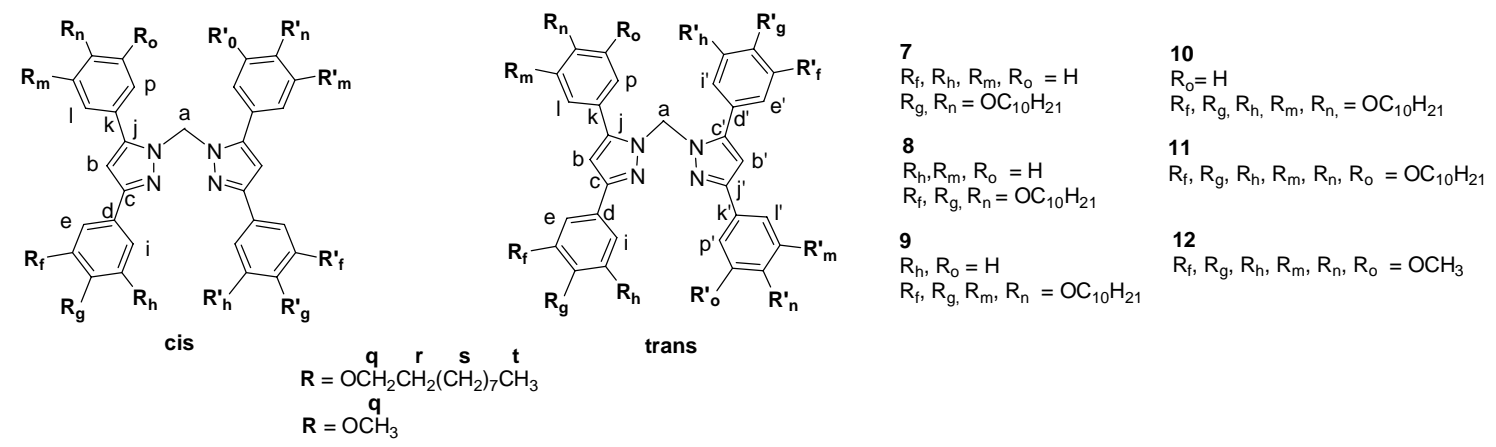

Bis[3,5-bis(4'-decyloxyphenyl)pyrazole-1-yl]methane 7: Eluent: hexane/ $\mathrm{CH}_{2} \mathrm{Cl}_{2}$ (2/3). Yield: $70 \%$. Anal. Calcd for $\mathrm{C}_{71} \mathrm{H}_{104} \mathrm{~N}_{4} \mathrm{O}_{4}$ : C 79.13, H 9.73, N 5.20. Found: C 79.17, H 9.68, N 5.16. IR (KBr): 1612, 1496 (arC-C), $1248(\mathrm{C}-\mathrm{O}) \mathrm{cm}^{-1} .{ }^{1} \mathrm{H}$ NMR (300 MHz, CDCl $\left.{ }_{3}\right) \delta 7.87\left(\mathrm{AA}^{\prime} \mathrm{BB}^{\prime}, 4 \mathrm{H}_{\mathrm{l}, \mathrm{p}}\right), 7.73$

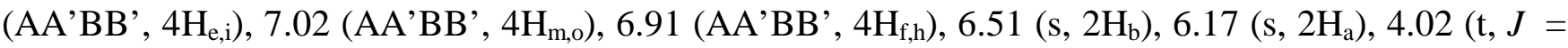
$\left.6.6 \mathrm{~Hz}, 4 \mathrm{H}_{\mathrm{q}}\right), 3.98\left(\mathrm{t}, J=6.6 \mathrm{~Hz}, 4 \mathrm{H}_{\mathrm{q}}\right), 1.85-1.77\left(\mathrm{~m}, 8 \mathrm{H}_{\mathrm{r}}\right), 1.49-1.39\left(\mathrm{~m}, 56 \mathrm{H}_{\mathrm{s}}\right), 0.89(\mathrm{t}, J=6.6 \mathrm{~Hz}$, $\left.12 \mathrm{H}_{\mathrm{t}}\right)$ ppm. ${ }^{13} \mathrm{C} \mathrm{NMR}\left(400 \mathrm{MHz}, \mathrm{CDCl}_{3}\right) \delta(159.6,159.0)_{\mathrm{n}},(151.3,146.3)_{\mathrm{c}, \mathrm{j}},(131.0,126.8)_{\mathrm{e}, \mathrm{i}, 1, \mathrm{p}},(125.9$, $122.4)_{\mathrm{d}, \mathrm{k}},(114.6,114.5)_{\mathrm{f}, \mathrm{h}, \mathrm{m}, \mathrm{o}}, 103.0_{\mathrm{b}},(68.1,68.0)_{\mathrm{q}}, 60.0_{\mathrm{a}},(31.9,29.7,29.6,29.4,29.3,26.1,22.7)_{\mathrm{r}, \mathrm{s}}$, 14.1 $\cdot \mathrm{ppm} . \mathrm{MS}(\mathrm{FAB}+) \mathrm{m} / z: 1078[\mathrm{M}+\mathrm{H}]^{+}$ 
hexane $/ \mathrm{CH}_{2} \mathrm{Cl}_{2}$ (9/1). Yield: $45 \%$. Anal. Calcd for $\mathrm{C}_{91} \mathrm{H}_{144} \mathrm{~N}_{4} \mathrm{O}_{6}$ : C 78.62, $\mathrm{H}$ 10.44, N 4.03. Found: $\mathrm{C}$ 78.49, H 10.56, N 4.02 . IR (KBr): 1615, 1503 (arC-C), 1245 (C-O) $\mathrm{cm}^{-1} .{ }^{1} \mathrm{H}$ NMR (300 MHz, $\mathrm{CDCl}_{3}$ ) $\delta 7.92\left(\mathrm{AA}^{\prime} \mathrm{BB}^{\prime}, 4 \mathrm{Hc}_{\mathrm{l}, \mathrm{p}} \mathrm{t}_{\mathrm{t}, \mathrm{p}}\right), 7.77\left(\mathrm{AA}^{\prime} \mathrm{BB}^{\prime}, 2 \mathrm{Ht}_{\mathrm{l}^{\prime}, \mathrm{p}^{\mathrm{p}}}\right), 7.76\left(\mathrm{~d}, J=1.6 \mathrm{~Hz}, 1 \mathrm{Ht}_{\mathrm{e}}{ }^{\prime}\right), 7.46(\mathrm{~d}, J=1.6 \mathrm{~Hz}$, $\left.1 \mathrm{Hc}_{\mathrm{e}}\right), 7.42\left(\mathrm{dd}, J=8.4 \mathrm{~Hz} J=2.0 \mathrm{~Hz}, 1 \mathrm{Ht}_{\mathrm{i}}{ }^{\prime}\right), 7.39\left(\mathrm{~d}, J=2.0 \mathrm{~Hz}, 1 \mathrm{Ht}_{\mathrm{e}}\right), 7.34(\mathrm{dd}, J=8.0 \mathrm{~Hz} J=1.6 \mathrm{~Hz}$, $\left.1 \mathrm{Ht}_{\mathrm{i}}\right), 7.42\left(\mathrm{dd}, J=8.4 \mathrm{~Hz}, J=2.0 \mathrm{~Hz}, 1 \mathrm{Hc}_{\mathrm{i}}\right), 7.04\left(\mathrm{AA}^{\prime} \mathrm{BB}^{\prime}, 2 \mathrm{Ht}_{\mathrm{m}, \mathrm{o}}\right), 7.02\left(\mathrm{AA}^{\prime} \mathrm{BB}^{\prime}, 2 \mathrm{Hc}_{\mathrm{m}, \mathrm{o}}\right), 6.97(\mathrm{~d}, J$ $\left.=8.4 \mathrm{~Hz}, 1 \mathrm{Ht}_{\mathrm{h}^{\prime}}\right), 6.93\left(\mathrm{AA}^{\prime} \mathrm{BB}^{\prime}, 2 \mathrm{Ht}_{\mathrm{m}^{\prime}, 0^{\prime}}\right), 6.90\left(\mathrm{~d}, J=8.0 \mathrm{~Hz}, 1 \mathrm{Hc}_{\mathrm{h}}\right), 6.88\left(\mathrm{~d}, J=8.4 \mathrm{~Hz}, 1 \mathrm{Ht}_{\mathrm{h}}\right), 6.55(\mathrm{~s}$, $\left.1 \mathrm{Ht}_{\mathrm{b}}\right), 6.52\left(\mathrm{~s}, 2 \mathrm{Hc}_{\mathrm{b}} \mathrm{t}_{\mathrm{b}}\right), 6.20\left(\mathrm{~s}, 2 \mathrm{Ht}_{\mathrm{a}}\right), 6.18\left(\mathrm{~s}, 1 \mathrm{Hc}_{\mathrm{a}}\right), 4.11-3.98\left(\mathrm{~m}, 18 \mathrm{Hc}, \mathrm{t}_{\mathrm{q}}\right), 1.90-1.74\left(\mathrm{~m}, 18 \mathrm{Hc}, \mathrm{t}_{\mathrm{r}}\right)$, $1.30-1.50\left(\mathrm{~m}, 126 \mathrm{Hc}, \mathrm{t}_{\mathrm{s}}\right), 0.91\left(\mathrm{t}, J=6.8 \mathrm{~Hz}, 27 \mathrm{Hc}, \mathrm{t}_{\mathrm{t}}\right) \mathrm{ppm} .{ }^{13} \mathrm{C} \mathrm{NMR}\left(400 \mathrm{MHz}, \mathrm{CDCl}_{3}\right) \delta(159.7$, $159.1)_{\mathrm{n}},(151.8,151.5)_{\mathrm{c}, \mathrm{j}},(149.6,149.23,149.20,149.18,149.1)_{\mathrm{f}, \mathrm{g}},(146.6,146.5,146,5)_{\mathrm{c}, \mathrm{j}},(131.0$, $126.9)_{\mathrm{l}, \mathrm{p}},(126.6,126.5,125.9)_{\mathrm{d}, \mathrm{k}}, 122.9_{\mathrm{e}},(122.4,122.4,122.2)_{\mathrm{d}, \mathrm{k}},(118.7,118.4,115.0,114.9,114.6$, $114.5,113.8,113.5)_{\mathrm{h}, \mathrm{m}, \mathrm{o}},(111.8,111.2)_{\mathrm{i},},(103.3,103.1,102.8)_{\mathrm{b}},(69.4,69.3,69.3,69.2,68.1,68.0)_{\mathrm{q}}$, $65.18_{\mathrm{a}},(32.0,31.9,29.7,29.7,29.6,29.6,29.5,29.5,29.4,29.4,29.3,26.2,26.1,26.0,22.7)_{\mathrm{r}, \mathrm{s}},(15.5$, 14.1)t. ppm. MS (MALDI+) m/z: $1391[\mathrm{M}+\mathrm{H}]^{+}$.

Bis[3,5-bis(3',4'-didecyloxyphenyl)pyrazole-1-yl]methane 9: Eluent: $\mathrm{CH}_{2} \mathrm{Cl}_{2}$. Yield: $35 \%$. Anal. Calcd for $\mathrm{C}_{111} \mathrm{H}_{184} \mathrm{~N}_{4} \mathrm{O}_{8}$ : C 78.30, H 10.89, N 3.29. Found: C 78.01, H 10.82, N 3.31 . IR (KBr): 1612, 1495 (arC-C), $1255(\mathrm{C}-\mathrm{O}) \mathrm{cm}^{-1} .{ }^{1} \mathrm{H}-\mathrm{NMR}\left(400 \mathrm{MHz}, \mathrm{CDCl}_{3}\right) \delta 7.74\left(\mathrm{~s}, 2 \mathrm{H}_{1}\right), 7.36(\mathrm{dd}, J=8 \mathrm{~Hz}, J=1.6$ $\left.\mathrm{Hz}, 2 \mathrm{H}_{\mathrm{p}}\right), 7.31\left(\mathrm{~s}, 2 \mathrm{H}_{\mathrm{e}}\right) 7.26\left(\mathrm{dd}, J=8.4 \mathrm{~Hz}, J=2.0 \mathrm{~Hz}, 2 \mathrm{H}_{\mathrm{i}}\right), 6.88\left(\mathrm{~d}, J=8.4 \mathrm{~Hz}, 2 \mathrm{H}_{\mathrm{o}}\right), 6.79(\mathrm{~d}, J=8$ $\left.\mathrm{Hz}, 2 \mathrm{H}_{\mathrm{h}}\right), 6.46\left(\mathrm{~s}, 2 \mathrm{H}_{\mathrm{b}}\right), 6.11\left(\mathrm{~s}, 2 \mathrm{H}_{\mathrm{a}}\right), 3.96\left(\mathrm{~m}, 16 \mathrm{H}_{\mathrm{q}}\right), 1.62-1.79\left(\mathrm{~m}, 16 \mathrm{H}_{\mathrm{r}}\right), 1.16-1.48\left(\mathrm{~m}, 112 \mathrm{H}_{\mathrm{s}}\right), 0.80$ $\left(\mathrm{m}, 24 \mathrm{H}_{\mathrm{t}}\right)$ ppm. ${ }^{13} \mathrm{C}-\mathrm{NMR}\left(100 \mathrm{MHz}, \mathrm{CDCl}_{3}\right) \delta 151.8_{\mathrm{j}},(149.6,149.2)_{\mathrm{f}, \mathrm{g}, \mathrm{m}, \mathrm{n}}, 146.7_{\mathrm{c}},(126.6,122.8)_{\mathrm{d}, \mathrm{k}}$, $(122.2,118.7,115.0,113.8,113.5,111.8)_{\mathrm{e}, \mathrm{h}, \mathrm{i}, \mathrm{l}, \mathrm{p}, \mathrm{p}}, 103.0_{\mathrm{b},}(69.3,69.3)_{\mathrm{q}}, 65.1_{\mathrm{a}},(31.9,29.7,29.6,29.5$, 29.4, 29.3, 26.1, 26.1, 26.1, 22.7) r, $, 14.1_{\mathrm{t}} . \mathrm{ppm} . \mathrm{MS}(\mathrm{FAB}+) \mathrm{m} / \mathrm{z}: 1702[\mathrm{M}]^{+}$. 
Bis[3-(3',4',5'-tridecyloxyphenyl)-5-(3',,4',-didecyloxyphenyl)pyrazole-1-yl]methane 10: Eluent: hexane/ $\mathrm{CH}_{2} \mathrm{Cl}_{2}$ (9/1). Yield: $20 \%$. Anal. Calcd for $\mathrm{C}_{131} \mathrm{H}_{224} \mathrm{~N}_{4} \mathrm{O}_{10}$ : C 78.08, H 11.20, N 2.78. Found: C78.15, H 11.39, N 2.79. IR (nujol, NaCl): 1612, 1498 (arC-C), $1253(\mathrm{C}-\mathrm{O}) \mathrm{cm}^{-1} .{ }^{1} \mathrm{H}$ NMR $(300 \mathrm{MHz}$, $\left.\mathrm{CDCl}_{3}\right) \delta 7.83(\mathrm{~d}, J=1.6 \mathrm{~Hz}, 1 \mathrm{Ht}), 7.77\left(\mathrm{~d}, J=1.6 \mathrm{~Hz}, 1 \mathrm{Hc}_{1}\right), 7.48\left(\mathrm{dd}, J=8 \mathrm{~Hz}, J=2.0 \mathrm{~Hz}, 1 \mathrm{Ht}_{\mathrm{p}}\right)$, $7.43\left(\mathrm{dd}, J=8.4 \mathrm{~Hz}, J=2.0 \mathrm{~Hz}, 1 \mathrm{Hc}_{\mathrm{p}}\right), 7.42\left(\mathrm{~d}, J=2 \mathrm{~Hz}, 1 \mathrm{Ht}_{12}\right), 7.36\left(\mathrm{~m}, 3 \mathrm{Ht}_{5}, 9^{\prime}, 16^{\prime}\right), 7.02\left(\mathrm{~s}, 2 \mathrm{Hc}_{5}\right)$, $6.99\left(\mathrm{~s}, 2 \mathrm{Ht}_{5,9}\right), 6.96\left(\mathrm{~d}, \mathrm{~J}=8.4 \mathrm{~Hz}, 2 \mathrm{Ht}_{\mathrm{o}}\right), 6.95\left(\mathrm{~d}, \mathrm{~J}=8.4 \mathrm{~Hz}, 1 \mathrm{Hc}_{\mathrm{o}}\right), 6.88\left(\mathrm{~d}, J=8.4 \mathrm{~Hz}, 1 \mathrm{Ht}_{\mathrm{o}^{\prime}}\right), 6.56(\mathrm{~s}$, $\left.1 \mathrm{Ht}_{\mathrm{b}}\right), 6.53\left(\mathrm{~s}, 1 \mathrm{Hc}_{\mathrm{b}}\right), 6.52\left(\mathrm{~s}, 1 \mathrm{Ht}_{\mathrm{b}}\right), 6.20\left(\mathrm{~s}, 3 \mathrm{Hc}_{\mathrm{a}}, \mathrm{t}_{\mathrm{a}}\right), 4.01\left(\mathrm{~m}, 40 \mathrm{H}_{\mathrm{q}}\right), 1.66-1.89\left(\mathrm{~m}, 40 \mathrm{H}_{\mathrm{r}}\right), 1.22-1.53$ $\left(\mathrm{m}, 280 \mathrm{H}_{\mathrm{s}}\right), 0.80\left(\mathrm{~m}, 60 \mathrm{H}_{\mathrm{t}}\right) \mathrm{ppm} .{ }^{13} \mathrm{C} \mathrm{NMR}\left(100 \mathrm{MHz}, \mathrm{CDCl}_{3}\right) \delta(153.3,153.2)_{\mathrm{f}, \mathrm{h}},(152.2,151.9$, $151.8)_{\mathrm{c}, \mathrm{j}},(149.6,149.2)_{\mathrm{m}, \mathrm{n}},(146.9,146.86,146.8)_{\mathrm{c}, \mathrm{j}},(138.5,138.4,138.36)_{\mathrm{g}},(128.6,128.5,126.4$, $124.8,122.6)_{\mathrm{d}, \mathrm{k}},(122.2,118.6,114.9,113.7,113.5,111.8,108.0)_{\mathrm{j}, \mathrm{o}, \mathrm{p}},(105.0,104.7)_{\mathrm{e}, \mathrm{i}},(103.2,102.8)_{\mathrm{b}}$, $(73.4)_{\mathrm{q}},(69.3,69.2,69.17)_{\mathrm{q}}, 59.9_{\mathrm{a}},(31.9,30.3,29.7,29.6,29.58,29.5,29.4,29.3,29.26,26.2,26.0,25.9$, 22.7) $)_{\mathrm{r}, \mathrm{s}}, 14.1_{\mathrm{t}} \cdot \mathrm{ppm} . \mathrm{MS}(\mathrm{MALDI}+) \mathrm{m} / z: 2014[\mathrm{M}]^{+}, 1014\left[\mathrm{M}-\mathrm{L}_{2,3}\right]^{+}$.

Bis[3,5-bis-(3',4',5'-tridecyloxyphenyl)pyrazole-1-yl]methane 11: Eluent: $\mathrm{CH}_{2} \mathrm{Cl}_{2}$. Yield: 39\%. Anal. Calcd for $\mathrm{C}_{151} \mathrm{H}_{264} \mathrm{~N}_{4} \mathrm{O}_{12}$ : C 77.91, H 11.43, N 2.39. Found: C 77.89, H 11.60, N 2.39. IR (nujol, NaCl): 1611, 1498 (arC-C), $1248(\mathrm{C}-\mathrm{O}) \mathrm{cm}^{-1} .{ }^{1} \mathrm{H}$ NMR (300 MHz, CDCl $\left.{ }_{3}\right) \delta 7.33\left(\mathrm{~s}, 4 \mathrm{H}_{1, \mathrm{p}}\right), 7.00\left(\mathrm{~s}, 4 \mathrm{H}_{\mathrm{e}, \mathrm{i}}\right), 6.53$ $\left(\mathrm{s}, 2 \mathrm{H}_{\mathrm{b}}\right), 6.22\left(\mathrm{~s}, 2 \mathrm{H}_{\mathrm{a}}\right), 4.00\left(\mathrm{~m}, 24 \mathrm{H}_{\mathrm{q}}\right), 1.66-1.84\left(\mathrm{~m}, 24 \mathrm{H}_{\mathrm{r}}\right), 1.24-1.47\left(\mathrm{~m}, 168 \mathrm{H}_{\mathrm{s}}\right), 0.86\left(\mathrm{~m}, 36 \mathrm{H}_{\mathrm{t}}\right) \mathrm{ppm}$. ${ }^{13} \mathrm{C}$ NMR $\left(100 \mathrm{MHz}, \mathrm{CDCl}_{3}\right) \delta(153.4,153.3)_{\mathrm{f}, \mathrm{g}, \mathrm{m}, \mathrm{o}}, 152.2_{\mathrm{j}}, 147.1_{\mathrm{c}}, 138.6_{\mathrm{g}, \mathrm{n}},(128.5,124.7)_{\mathrm{d}, \mathrm{k}},(108.0$, $105.0)_{\mathrm{e}, \mathrm{i}, \mathrm{l}, \mathrm{p}}, 103.2_{\mathrm{b}},(73.5,69.4,69.3)_{\mathrm{q}}, 53.4_{\mathrm{a}},(31.9,30.4,29.8,29.7,29.6,29.55,29.5,29.4,26.2,26.1$, 22.7) $)_{\mathrm{r}, \mathrm{s}}, 14.1_{\mathrm{t}} \mathrm{ppm}$. MS (MALDI+) $\mathrm{m} / z: 2328[\mathrm{M}+\mathrm{H}]^{+}$.

Bis[3,5-bis-(3',4',5'-trimethoxyphenyl)pyrazole-1-yl]methane 12: Eluent: $\mathrm{CH}_{2} \mathrm{Cl}_{2}$. Yield: 33\%. Anal. Calcd for $\mathrm{C}_{43} \mathrm{H}_{48} \mathrm{~N}_{4} \mathrm{O}_{12}$ : C 63.54, H 5.95, N 6.89. Found: C 63.33, H 6.21, N 6.89. IR (nujol, NaCl): 1614, 1507 (arC-C), $1253(\mathrm{C}-\mathrm{O}) \mathrm{cm}^{-1} .{ }^{1} \mathrm{H}$ NMR (300 MHz, $\left.\mathrm{CDCl}_{3}\right) \delta 77.32\left(\mathrm{~s}, 4 \mathrm{H}_{1}\right), 7.04\left(\mathrm{~s}, 4 \mathrm{H}_{\mathrm{e}}\right), 6.60$ $\left(\mathrm{s}, 2 \mathrm{H}_{\mathrm{b}}\right), 6.31\left(\mathrm{~s}, 2 \mathrm{H}_{\mathrm{a}}\right), 3.91\left(\mathrm{~s}, 18 \mathrm{H}_{\mathrm{q}}\right), 3.90\left(\mathrm{~s}, 12 \mathrm{H}_{\mathrm{q}}\right), 3.87\left(\mathrm{~s}, 6 \mathrm{H}_{\mathrm{q}}\right) \mathrm{ppm} .{ }^{13} \mathrm{C} \mathrm{NMR}\left(100 \mathrm{MHz}, \mathrm{CDCl}_{3}\right) \delta$ 
$(153.5,153.4)_{\mathrm{f}, \mathrm{m}}, 152.1_{\mathrm{i}}, 146.9_{\mathrm{c}}, 138.7_{\mathrm{g}, \mathrm{n}}, 128.8_{\mathrm{k}}, 125.2_{\mathrm{d}}, 107.0_{\mathrm{l}}, 103.6_{\mathrm{b}}, 103.3_{\mathrm{e}}, 60.9_{\mathrm{q}}, 56.5_{\mathrm{q}}, 56.2_{\mathrm{a}} \mathrm{ppm}$.

MS (MALDI+) $m / z: 813[\mathrm{M}+\mathrm{H}]$

\section{General procedure for the synthesis of the complexes $\left[\mathrm{ZnCl}_{2}(\mathrm{Hpz})_{2}\right]$ and $\left[\mathrm{ZnCl}_{2}(\mathrm{Bpzm}](13-24)\right.$ :}

A solution of anhydrous $\mathrm{ZnCl}_{2}$ in THF (2 ml) was placed in a Schlenk tube containing a solution of the Hpz ligand (10 mmol) or, alternatively, a solution of the Bpzm ligand (5 mmol). The reaction mixture was degassed using a vacuum line, stirred at room temperature for $1 \mathrm{~h}$ and the solvent was then evaporated. The complexes were isolated by precipitation with hexane. In cases where the complexes were soluble in hexane they were dissolved in hexane, filtered and evaporated to dryness.

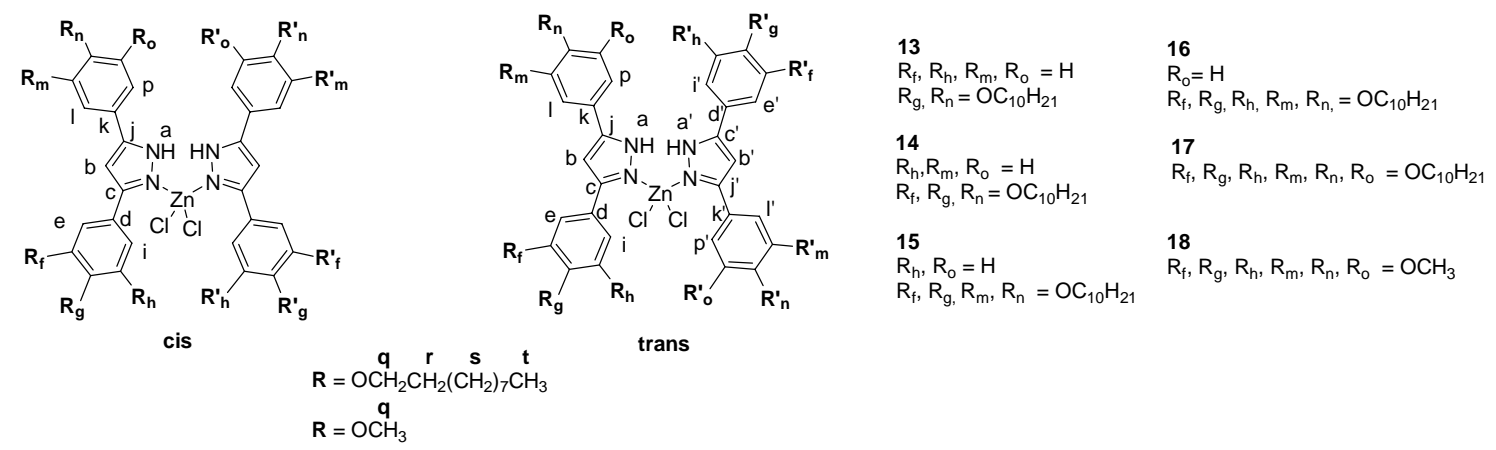

Dichlorobis(3-(4'-decyloxyphenyl)-5-(4'’-decyloxyphenyl)-1H-pyrazole)zinc(II) 13: Precipitation with hexane. Yield: > 90\%. Anal. Calcd for $\mathrm{C}_{70} \mathrm{H}_{104} \mathrm{Cl}_{2} \mathrm{~N}_{4} \mathrm{O}_{4} \mathrm{Zn}$ : C 69.95; H 8.72; N 4.66. Found: C 69.87; $\mathrm{H}$ 8.70; N 4.60. IR (KBr): $3303(\mathrm{~N}-\mathrm{H}), 1611,1500$ (arC-C), $1256(\mathrm{C}-\mathrm{O}) \mathrm{cm}^{-1} .{ }^{1} \mathrm{H}$ NMR (300 MHz, dmsod $\mathrm{d}_{6} \delta 7.75\left(\mathrm{AA}^{\prime} \mathrm{BB}^{\prime}, 4 \mathrm{H}_{\mathrm{l}, \mathrm{p}}\right), 7.69\left(\mathrm{~A}^{\prime} \mathrm{BB}^{\prime}, 4 \mathrm{H}_{\mathrm{e}, \mathrm{i}}\right), 7.01\left(\mathrm{AA}^{\prime} \mathrm{BB}^{\prime}, 4 \mathrm{H}_{\mathrm{m}, \mathrm{o}}\right), 6.96\left(\mathrm{AA}^{\prime} \mathrm{BB}^{\prime}, 4 \mathrm{H}_{\mathrm{f}, \mathrm{h}}\right), 6.95(\mathrm{~s}$, $\left.2 \mathrm{H}_{\mathrm{b}}\right), 4.00\left(\mathrm{t}, J=6.3 \mathrm{~Hz}, 4 \mathrm{H}_{\mathrm{q}}\right), 3.98\left(\mathrm{t}, J=6.3 \mathrm{~Hz}, 4 \mathrm{H}_{\mathrm{q}}\right), 1.76-1.68\left(\mathrm{~m}, 8 \mathrm{H}_{\mathrm{r}}\right), 1.42-1.20\left(\mathrm{~m}, 56 \mathrm{H}_{\mathrm{s}}\right), 0.86$ $\left(\mathrm{t}, J=6.3 \mathrm{~Hz}, 12 \mathrm{H}_{\mathrm{t}}\right) \mathrm{ppm} .{ }^{13} \mathrm{C}$ NMR non-soluble. MS (FAB+) $m / z: 1164[\mathrm{M}-\mathrm{Cl}]^{+}$. 
Dichlorobis(3-(3',4'-didecyloxyphenyl)-5-(4''-decyloxyphenyl)-1H-pyrazole)zinc(II) 14: Precipitation with hexane. Yield: > 90\%. Anal. Calcd for $\mathrm{C}_{90} \mathrm{H}_{144} \mathrm{Cl}_{2} \mathrm{~N}_{4} \mathrm{O}_{6} \mathrm{Zn}$ : C 71.38, H 9.58, N 3.70. Found: $\mathrm{C}$ 71.02, H 9.58, N 3.69. IR (KBr): 3293 (N-H), 1614, 1504(arC-C), 1255(C-O) $\mathrm{cm}^{-1} .{ }^{1} \mathrm{H}$ NMR (400 MHz, $\left.\mathrm{CDCl}_{3}\right) \delta 11.40\left(\mathrm{~s}(\mathrm{~b}), 2 \mathrm{H}_{\mathrm{a}}\right), 7.36\left(\mathrm{~s}(\mathrm{~b}), 4 \mathrm{H}_{\mathrm{l}, \mathrm{p}}\right), 7.05-6.95\left(\mathrm{~m}, 8 \mathrm{H}_{\mathrm{e}, \mathrm{h}, \mathrm{m}, \mathrm{o}}\right), 6.64\left(\mathrm{~s}(\mathrm{~b}), 2 \mathrm{H}_{\mathrm{i}}\right), 6.31\left(\mathrm{~s}, 2 \mathrm{H}_{\mathrm{b}}\right)$, $4.00\left(\mathrm{~s}(\mathrm{~b}), 8 \mathrm{H}_{\mathrm{q}}\right), 3.70-3.54\left(\mathrm{~m}, 4 \mathrm{H}_{\mathrm{q}}\right), 1.82\left(\mathrm{~m}, 12 \mathrm{H}_{\mathrm{r}}\right), 1.48-1.17\left(\mathrm{~m}, 84 \mathrm{H}_{\mathrm{s}}\right), 0.83-0.79\left(\mathrm{~m}, 18 \mathrm{H}_{\mathrm{t}}\right) \mathrm{ppm} .{ }^{13} \mathrm{C}$ NMR (75 MHz, $\left.\mathrm{CDCl}_{3}\right) \delta 160.7$ (b), 154.4 (b), 153.9 (b), 152.9 (b), 145.2 (b), 138.7 (b), 128.6 (b), 127.3 (b), 124.9 (b), 119.4 (b), 115.2 (b), 113.8 (b), 105.5 (b), 104.6, 101.8, 77.4, 77.2, 77.0, 76.6, 73.4, $69.3,68.2,32.0,31.9,30.4,29.8,29.7,29.6,29.56,29.5,29.4,29.35,29.2,26.2,26.0,22.72,22.7,14.1$ ppm. MS (MALDI+) m/z: 1477 [M-Cl].

Dichlorobis(3,5-bis(3'4'-didecyloxyphenyl)-1H-pyrazole)zinc(II) 15: Precipitation with hexane. Yield: > 90\%. Anal. Calcd for $\mathrm{C}_{110} \mathrm{H}_{184} \mathrm{Cl}_{2} \mathrm{~N}_{4} \mathrm{O}_{8} \mathrm{Zn}$ : C 72.32, H 10.15, N 3.07. Found: C 72.29, H 10.13, N 3.08. IR (KBr): $3298(\mathrm{~N}-\mathrm{H}), 1611,1513$ (arC-C), $1264(\mathrm{C}-\mathrm{O}) \mathrm{cm}^{-1} .{ }^{1} \mathrm{H}$ NMR (400 MHz, $\left.\mathrm{CDCl}_{3}\right) \delta$ $11.36\left(\mathrm{~s}, 2 \mathrm{H}_{\mathrm{a}}\right), 7.08\left(\mathrm{~m}, 2 \mathrm{H}_{\mathrm{h}}\right), 6.99\left(\mathrm{~m}, 4 \mathrm{H}_{\mathrm{e}, \mathrm{l}}\right), 6.92\left(\mathrm{~m}, 4 \mathrm{H}_{\mathrm{i}, \mathrm{o}}\right), 6.57\left(\mathrm{~m}, 2 \mathrm{H}_{\mathrm{p}}\right), 6.33\left(\mathrm{~s}, 2 \mathrm{H}_{\mathrm{b}}\right), 4.09-4.02(\mathrm{~m}$, $\left.4 \mathrm{H}_{\mathrm{q}}\right), 3.96-3.88\left(\mathrm{~m}, 4 \mathrm{H}_{\mathrm{q}}\right), 3.77-3.73\left(\mathrm{~m}, 4 \mathrm{H}_{\mathrm{q}}\right), 3.71-3.65\left(\mathrm{~m}, 4 \mathrm{H}_{\mathrm{q}}\right), 1.91-1.82\left(\mathrm{~m}, 12 \mathrm{H}_{\mathrm{r}}\right), 1.75-1.64(\mathrm{~m}$, $\left.4 \mathrm{H}_{\mathrm{r}}\right), 1.56-1.45\left(\mathrm{~m}, 8 \mathrm{H}_{\mathrm{s}}\right), 1.43-1.23\left(\mathrm{~m}, 104 \mathrm{H}_{\mathrm{s}}\right), 0.90-0.87\left(\mathrm{~m}, 24 \mathrm{H}_{\mathrm{t}}\right) \mathrm{ppm} .{ }^{13} \mathrm{C} \mathrm{NMR}\left(75 \mathrm{MHz}, \mathrm{CDCl}_{3}\right) \delta$ 154.1 (b), 150.1 (b), 149.5 (b), 145.4 (b), 122.7 (b), 120.1 (b), 118.9 (b), 113.0 (b), 111.3 (b), 101.7, $69.5,69.1,68.0,32.2,31.9,31.7,31.0,29.7,29.7,29.6,29.5,29.4,29.2,26.1,26.0,25.6,22.9,22.7$, 21.2, 17.0, 16.8, $14.1 \mathrm{ppm}$. MS (FAB+) m/z: 1789 [M-Cl], $1754\left[\mathrm{M}^{-\mathrm{Cl}_{2}}\right], 845\left[\mathrm{~L}_{2,2}{ }^{+\cdot}\right]$.

Dichlorobis(3-(3',4',5'-tridecyloxyphenyl)-5-(3', 4''-didecyloxyphenyl)-1H-pyrazole)zinc(II) $\quad \mathbf{1 6 :}$

Disolved in hexane. Yield: > 90\%. Anal. Calcd for $\mathrm{C}_{130} \mathrm{H}_{224} \mathrm{Cl}_{2} \mathrm{~N}_{4} \mathrm{O}_{10} \mathrm{Zn}$ : C 72.98, H 10.55, N 2.62. Found: C 72.75, H 10.49, N 2.66. IR (thin film): 3296(N-H), 1587, $1503(\operatorname{arC}-\mathrm{C}), 1257(\mathrm{C}-\mathrm{O}) \mathrm{cm}^{-1} .{ }^{1} \mathrm{H}$ NMR (300 MHz, $\left.\mathrm{CDCl}_{3}\right) \delta 11.46\left(\mathrm{~s}(\mathrm{~b}), 2 \mathrm{H}_{\mathrm{a}}\right), 7.11\left(\mathrm{~m}, 1 \mathrm{H}_{\mathrm{h}}\right), 7.03\left(\mathrm{~s}(\mathrm{~b}), 1 \mathrm{H}_{\mathrm{e}}\right), 6.93\left(\mathrm{~m}, 1 \mathrm{H}_{\mathrm{h}^{\prime}}\right), 6.89(\mathrm{~s}(\mathrm{~b})$, 
$\left.1 \mathrm{H}_{\mathrm{i}}\right), 6.69\left(\mathrm{~s}(\mathrm{~b}), 1 \mathrm{H}_{\mathrm{e}^{\prime}}\right), 6.56\left(\mathrm{~s}(\mathrm{~b}), 1 \mathrm{H}_{\mathrm{i}}{ }^{\prime}\right), 6.50\left(\mathrm{~s}(\mathrm{~b}), 2 \mathrm{H}_{\mathrm{l}, \mathrm{p}}\right), 6.35\left(\mathrm{~s}(\mathrm{~b}), 2 \mathrm{H}_{\mathrm{b}}\right), 4.00\left(\mathrm{~m}, 8 \mathrm{H}_{\mathrm{q}}\right), 3.79(\mathrm{~m}$ ,12 $\left.\mathrm{H}_{\mathrm{q}}\right), 1.85\left(\mathrm{~m}, 8 \mathrm{H}_{\mathrm{r}}\right), 1.65\left(\mathrm{~m}, 12 \mathrm{H}_{\mathrm{r}}\right), 1.56-1.21\left(\mathrm{~m}, 140 \mathrm{H}_{\mathrm{s}}\right), 0.90-0.86\left(\mathrm{~m}, 30 \mathrm{H}_{\mathrm{t}}\right) \mathrm{ppm} .{ }^{13} \mathrm{C}$ NMR $(75$ $\left.\mathrm{MHz}, \mathrm{CDCl}_{3}\right) \delta 154.4$ (b), 153.8 (b), 153.0 (b), 151.0 (b), 149.7 (b), 149.0 (b), 145.6 (b), 140.0 (b), 138.6 (b), 124.9 (b), 122.2 (b), 120.2 (b), 119.7 (b), 119.0 (b), 113.4 (b), 112.9 (b), 111.3 (b), 105.3 (b), 104.8 (b), 101.9, 73.4, 69.6, 69.2, 32.0, 31.7, 31.4, 30.4, 30.2, 29.8, 29.7, 29.6, 29.5, 29.4, 29.2, 27.9, 27.3, 26.2, 26.11, 26.0, 25.5, 25.0, 24.1, 22.9, 22.7, 22.5, 21.4, 14.1 ppm. MS (FAB+) m/z: 2104 [M$\mathrm{Cl}]$.

Dichlorobis(3,5-bis(3'4',5'-tridecyloxyphenyl)-1H-pyrazole)zinc(II) 17: Disolved in hexane. Yield: > 90\%. Anal. Calcd for $\mathrm{C}_{150} \mathrm{H}_{264} \mathrm{Cl}_{2} \mathrm{~N}_{4} \mathrm{O}_{12} \mathrm{Zn}$ : C 73.47, H 10.85, N 2.28. Found: C 73.20, H 10.88, 2.25. IR (thin film): $3320(\mathrm{~N}-\mathrm{H}), 1586,1497$ (arC-C), $1241(\mathrm{C}-\mathrm{O}) \mathrm{cm}^{-1} .{ }^{1} \mathrm{H}$ NMR $\left(300 \mathrm{MHz}, \mathrm{CDCl}_{3}\right) \delta 11.46$ (s, $\left.2 \mathrm{H}_{\mathrm{a}}\right), 6.72\left(\mathrm{~s}, 4 \mathrm{H}_{\mathrm{e}, \mathrm{i}}\right), 6.46\left(\mathrm{~s}, 4 \mathrm{H}_{\mathrm{l}, \mathrm{p}}\right), 6.36\left(\mathrm{~s}, 2 \mathrm{H}_{\mathrm{b}}\right), 4.07-3.98\left(\mathrm{~m}, 16 \mathrm{H}_{\mathrm{q}, \mathrm{qpara}}\right), 3.84-3.81\left(\mathrm{~m}, 4 \mathrm{H}_{\mathrm{q}}\right), 3.78-$ $3.72\left(\mathrm{~m}, 4 \mathrm{H}_{\mathrm{q}}\right), 1.90-1.71\left(\mathrm{~m}, 16 \mathrm{H}_{\mathrm{r}}\right), 1.69-1.58\left(\mathrm{~m}, 20 \mathrm{H}_{\mathrm{r}, \mathrm{s}}\right), 1.52-1.46\left(\mathrm{~m}, 12 \mathrm{H}_{\mathrm{s}}\right), 1.21\left(\mathrm{~m}, 144 \mathrm{H}_{\mathrm{s}}\right), 0.89-$

$0.86\left(\mathrm{~m}, 36 \mathrm{H}_{\mathrm{t}}\right) \mathrm{ppm} .{ }^{13} \mathrm{C} \mathrm{NMR}\left(100 \mathrm{MHz}, \mathrm{CDCl}_{3}\right) \delta 153.4,152.9,151.9,144.9,139.1,137.6,123.9$, $121.1,104.1,103.8,101.4,72.5,68.5,68.4,68.1,31.0,29.4,28.8,28.7,28.6,28.5,28.48,28.4,25.2$, 21.7, 13.1 ppm. MS (MALDI+) $m / z: 1158\left[\mathrm{~L}_{3,3}+\mathrm{H}\right]$.

Dichlorobis(3,5-bis(3',4',5'-trimethoxyphenyl)-1H-pyrazole)zinc(II) 18: Disolved in hexane. Yield: > 90\%. Anal. Calcd for $\mathrm{C}_{42} \mathrm{H}_{48} \mathrm{Cl}_{2} \mathrm{~N}_{4} \mathrm{O}_{12} \mathrm{Zn}$ : C 53.83, H 5.16, N 5.98. Found: C 53.54, H 4.90, N 5.65 \%. IR (KBr): 3286, 3269, 3229, 3190, 3147, $3112(\mathrm{~N}-\mathrm{H},=\mathrm{C}-\mathrm{H}), 1593,1504(\operatorname{arC}-\mathrm{C}), 1237(\mathrm{C}-\mathrm{O}) \mathrm{cm}^{-1} .{ }^{1} \mathrm{H}$ NMR $\left(300 \mathrm{MHz}, \mathrm{CDCl}_{3}\right) \delta 6.76\left(\mathrm{~s}, 8 \mathrm{H}_{\mathrm{e}, 1}\right), 6.55\left(\mathrm{~s}, 2 \mathrm{H}_{\mathrm{b}}\right), 3.85\left(\mathrm{~s}, 12 \mathrm{H}_{\mathrm{q}}\right), 3.80\left(\mathrm{~s}, 24 \mathrm{H}_{\mathrm{q}}\right)$ ppm. ${ }^{13} \mathrm{C} \mathrm{NMR}$ $\left(100 \mathrm{MHz} \mathrm{CDCl}_{3}\right) \delta 153.4_{\mathrm{c}, \mathrm{f}, \mathrm{i}, \mathrm{m}}, 138.8_{\mathrm{g}, \mathrm{n}}, 124.9_{\mathrm{d}, \mathrm{k}}(\mathrm{b}), 103.4_{\mathrm{e}, \mathrm{l}}, 101.5_{\mathrm{b}}, 60.9_{\mathrm{q}}, 56.2_{\mathrm{q}} \mathrm{ppm} . \mathrm{MS}(\mathrm{MALDI}+)$ $m / z: 899$ [M-Cl] 


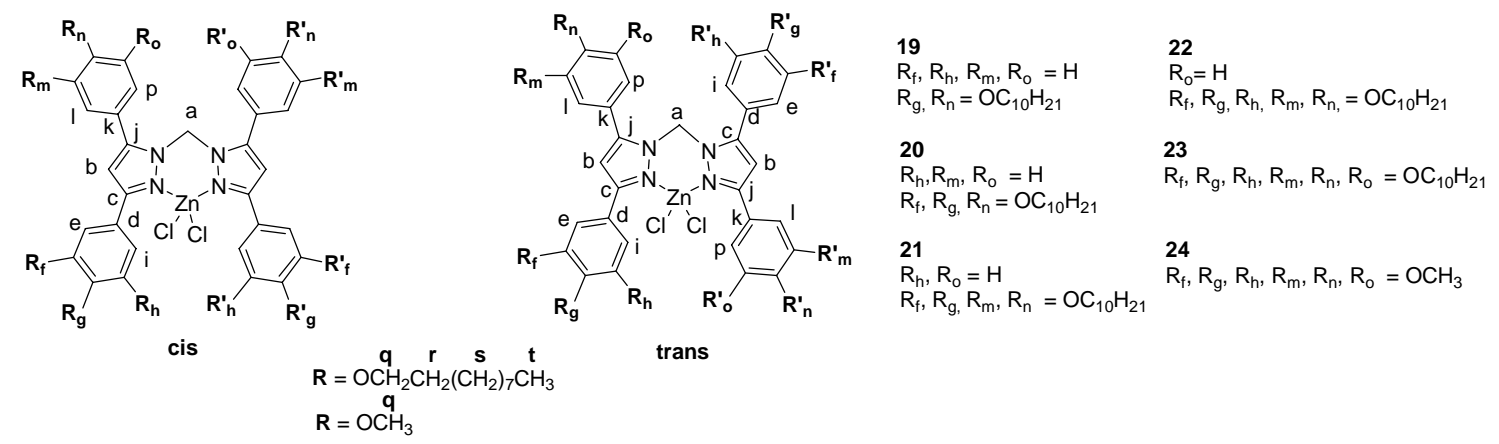

Dichloro[bis(3,5-bis(4'-decyloxyphenyl)pyrazole-1-yl)methane]zinc(II) 19: Precipitation with hexane. Yield: > 90\%. Anal. Calcd for $\mathrm{C}_{71} \mathrm{H}_{104} \mathrm{Cl}_{2} \mathrm{~N}_{4} \mathrm{O}_{4} \mathrm{Zn}$ : C 70.25, H 8.64, N 4.62. Found: C 70.24, H 8.65, N 4.58. IR (Nujol, $\mathrm{NaCl}): 3047\left(\mathrm{C}_{\mathrm{sp} 2}-\mathrm{H}\right), 1612,1578,1500(\mathrm{C}=\mathrm{N}, \operatorname{arC}-\mathrm{C}), 1250(\mathrm{C}-\mathrm{O}) \mathrm{cm}^{-1} .{ }^{1} \mathrm{H}$ NMR $(400$

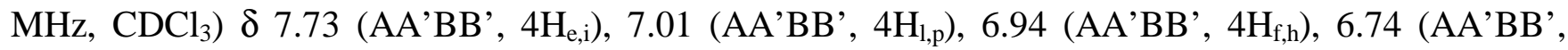
$\left.4 \mathrm{H}_{\mathrm{m}, \mathrm{o}}\right), 6.45\left(\mathrm{~s}, 4 \mathrm{H}_{\mathrm{a}, \mathrm{b}}\right), 3.94\left(\mathrm{t}, J=6.2 \mathrm{~Hz}, 4 \mathrm{H}_{\mathrm{q}}\right), 3.93\left(\mathrm{t}, J=6.2 \mathrm{~Hz}, 4 \mathrm{H}_{\mathrm{q}}\right), 1.86-1.70\left(\mathrm{~m}, 8 \mathrm{H}_{\mathrm{r}}\right), 1.49-1.25$ $\left(\mathrm{m}, 56 \mathrm{H}_{\mathrm{s}}\right), 0.90-0.85\left(\mathrm{~m}, 12 \mathrm{H}_{\mathrm{t}}\right) \mathrm{ppm} .{ }^{13} \mathrm{C} \mathrm{NMR}\left(100 \mathrm{MHz}, \mathrm{CDCl}_{3}\right) \delta(160.7,160.6)_{\mathrm{n}},(147.3,130.1)_{\mathrm{c}, \mathrm{j}}$ $(129.8,121.7)_{\mathrm{d}, \mathrm{k}},(118.9,115.2)_{\mathrm{e}, \mathrm{i}, \mathrm{l}, \mathrm{p}},(114.7,112.6)_{\mathrm{f}, \mathrm{h}, \mathrm{m}, \mathrm{o}}, 106.2_{\mathrm{b}},(68.3,68.0)_{\mathrm{q}}, 57.2_{\mathrm{a}},(31.9,29.5,29.3$, 26.0, 22.6) $)_{\mathrm{r}, \mathrm{s}}, 14.1_{\mathrm{t}} \mathrm{ppm} . \mathrm{MS}(\mathrm{FAB}+) \mathrm{m} / z: 1175[\mathrm{M}-\mathrm{Cl}]^{+}$.

Dissolved in hexane. Yield: > 90\%. Anal. Calcd for $\mathrm{C}_{91} \mathrm{H}_{144} \mathrm{Cl}_{2} \mathrm{~N}_{4} \mathrm{O}_{6} \mathrm{Zn}$ : C 71.60, $\mathrm{H}$ 9.51, $\mathrm{N}$ 3.67. Found: C 71.45, H 9.45, N 3.67. IR (KBr): 1611, 1502 (arC-C), 1264 (C-O) cm ${ }^{-1} .{ }^{1} \mathrm{H}$ NMR (400 MHz, $\left.\mathrm{CDCl}_{3}\right) \delta 7.75\left(\mathrm{AA}^{\prime} \mathrm{BB}, \mathrm{Ht}^{\prime}, \mathrm{p}^{\prime}\right), 7.46\left(\mathrm{~d}, J=2.0 \mathrm{~Hz}, 1 \mathrm{Hc}_{\mathrm{e}}\right), 7.41\left(\mathrm{~d}, J=2.0 \mathrm{~Hz}, 1 \mathrm{Ht}_{\mathrm{e}}\right), 7.30-7.26(\mathrm{~m}$, $\left.2 \mathrm{Hc}_{\mathrm{i}} \mathrm{t}_{\mathrm{i}}\right), 7.03\left(\mathrm{AA}^{\prime} \mathrm{BB}^{\prime}, 2 \mathrm{Hc}_{\mathrm{l}, \mathrm{p}}\right), 6.98\left(\mathrm{AA}^{\prime} \mathrm{BB}^{\prime}, 2 \mathrm{Ht}_{\mathrm{m}^{\prime}, \mathrm{o}^{\prime}}\right), 6.97\left(\mathrm{AA}^{\prime} \mathrm{BB} \mathrm{B}^{\prime}, 2 \mathrm{Ht}_{\mathrm{l}, \mathrm{p}}\right), 6.91(\mathrm{~d}, J=8.8 \mathrm{~Hz}$, $\left.\left.2 \mathrm{Hc}_{\mathrm{h}} \mathrm{t}_{\mathrm{h}}\right), 6.77\left(\mathrm{AA}^{\prime} \mathrm{BB}^{\prime}, 2 \mathrm{Hc}_{\mathrm{m}, \mathrm{o}}\right), 6.77\left(\mathrm{~d}, J=2.0 \mathrm{~Hz}, 1 \mathrm{Ht}_{\mathrm{e}}\right)^{\prime}\right), 6.72\left(\mathrm{AA}^{\prime} \mathrm{BB}^{\prime}, 2 \mathrm{Ht}_{\mathrm{m}, \mathrm{o}}\right), 6.63(\mathrm{~d}, J=8.0 \mathrm{~Hz}$, $\left.\left.1 \mathrm{Ht}_{\mathrm{h}}\right)\right), 6.53\left(\mathrm{dd}, J=8.0 \mathrm{~Hz}, J=2.0 \mathrm{~Hz}, 1 \mathrm{Ht}_{\mathrm{i}}{ }^{\prime}\right), 6.51\left(\mathrm{~s}, 2 \mathrm{Ht}_{\mathrm{a}}\right), 6.48\left(\mathrm{~s}, 2 \mathrm{Ht}_{\mathrm{b}} \mathrm{c}_{\mathrm{b}}\right), 6.46\left(\mathrm{~s}, 1 \mathrm{Ht}_{\mathrm{b}}{ }^{\prime}\right), 6.45(\mathrm{~s}$, $\left.1 \mathrm{Hc}_{\mathrm{a}}\right), 4.19\left(\mathrm{t}, J=6.4 \mathrm{~Hz}, 2 \mathrm{Hc}_{\mathrm{q}}\right), 4.17\left(\mathrm{t}, J=6.8 \mathrm{~Hz}, 2 \mathrm{Ht}_{\mathrm{q}}\right), 4.02-3.93\left(\mathrm{~m}, 12 \mathrm{Hc}, \mathrm{t}_{\mathrm{q}}\right), 1.90-1.75(\mathrm{~m}$, 
$\left.16 \mathrm{Hc}, \mathrm{t}_{\mathrm{r}}\right), 1.63-1.20\left(\mathrm{~m}, 126 \mathrm{Hc}, \mathrm{t}_{\mathrm{s}}\right), 0.88\left(\mathrm{~m}, 27 \mathrm{Hc}, \mathrm{t}_{\mathrm{t}}\right) \mathrm{ppm} .{ }^{13} \mathrm{C} \mathrm{NMR}\left(100 \mathrm{MHz}, \mathrm{CDCl}_{3}\right) \delta(164.5,160.6$, $160.5)_{\mathrm{n}},(156.5,156.46,156.3,156.2,150.8,150.7,149.6,149.2,147.4,147.3)_{\mathrm{c}, \mathrm{f}, \mathrm{g}, \mathrm{j}},(131.4,130.1$, $130.0,129.7)_{\mathrm{m}, \mathrm{o}},(122.1,122.07,121.7,121.4,120.9,120.8)_{\mathrm{d}, \mathrm{k}},(119.2,118.9,118.7,115.2,114.9$, $114.6,113.9,113.8,113.7,113.3,113.2)_{\mathrm{h}, \mathrm{i}, \mathrm{p}, \mathrm{p}}, 106.2_{\mathrm{b}},(69.6,69.5,69.4,69.1,69.0,68.2,68.17,67.9)_{\mathrm{a}, \mathrm{q}}$, $(31.9,31.86,31.8,29.7,29.6,29.5,29.4,29.35,29.3,29.29,29.26,29.2,29.1,26.1,26.05,26.03,26.0$, 25.97, 22.7) $)_{\mathrm{r}, \mathrm{s}}, 14.1_{\mathrm{t}} \mathrm{ppm} . \mathrm{MS}(\mathrm{MALDI}+) \mathrm{m} / z: 1489$ [M-Cl].

Dichloro[bis(3,5-bis(3',4'-didecyloxyphenyl)pyrazole-1-yl)methane]zinc(II) 21: Dissolved in hexane. Yield: > 90\%. Anal. Calcd for $\mathrm{C}_{111} \mathrm{H}_{184} \mathrm{Cl}_{2} \mathrm{~N}_{4} \mathrm{O}_{8} \mathrm{Zn}$ : C 72.50, H 10.09, N 3.05. Found: C 72.69, H 9.94, 2.99. IR (KBr): 1606,1505 (arC-C), $1270(\mathrm{C}-\mathrm{O}) \mathrm{cm}^{-1} .{ }^{1} \mathrm{H}$ NMR $\left(400 \mathrm{MHz}, \mathrm{CDCl}_{3}\right) \delta 7.46(\mathrm{~d}, J=2.0$ $\left.\mathrm{Hz}, 2 \mathrm{H}_{\mathrm{e}}\right), 7.26\left(\mathrm{dd}, J=8.4 \mathrm{~Hz}, J=2.0 \mathrm{~Hz}, 2 \mathrm{H}_{\mathrm{i}}\right), 6.89\left(\mathrm{~d}, J=8.4 \mathrm{~Hz}, 2 \mathrm{H}_{\mathrm{h}}\right), 6.76\left(\mathrm{~d}, J=1.6 \mathrm{~Hz}, 2 \mathrm{H}_{1}\right), 6.56$ $\left(\mathrm{d}, J=8.4 \mathrm{~Hz}, 2 \mathrm{H}_{\mathrm{o}}\right), 6.50\left(\mathrm{~s}, 2 \mathrm{H}_{\mathrm{a}}\right), 6.48\left(\mathrm{~s}, 2 \mathrm{H}_{\mathrm{b}}\right), 6.44\left(\mathrm{dd}, J=8.4 \mathrm{~Hz}, J=1.6 \mathrm{~Hz}, 2 \mathrm{H}_{\mathrm{p}}\right), 4.19(\mathrm{t}, J=6.4$ $\left.\mathrm{Hz}, 4 \mathrm{H}_{\mathrm{q}}\right), 4.00\left(\mathrm{t}, J=6.8 \mathrm{~Hz}, 4 \mathrm{H}_{\mathrm{q}}\right), 3.97\left(\mathrm{t}, J=6.8 \mathrm{~Hz}, 4 \mathrm{H}_{\mathrm{q}}\right), 3.93\left(\mathrm{t}, J=6.8 \mathrm{~Hz}, 4 \mathrm{H}_{\mathrm{q}}\right), 1.79-1.87(\mathrm{~m}$, $\left.16 \mathrm{H}_{\mathrm{r}}\right), 1.27-1.50\left(\mathrm{~m}, 112 \mathrm{H}_{\mathrm{s}}\right), 0.87\left(\mathrm{~m}, 24 \mathrm{H}_{\mathrm{t}}\right) \mathrm{ppm} .{ }^{13} \mathrm{C} \mathrm{NMR}\left(100 \mathrm{MHz}, \mathrm{CDCl}_{3}\right) \delta 155.4_{\mathrm{j}},(150.7,150.0$, $148.8,147.4,146.8)_{\mathrm{c}, \mathrm{f}, \mathrm{g}, \mathrm{m}, \mathrm{n}},(121.4,120.6,120.1,118.4)_{\mathrm{d}, \mathrm{i}, \mathrm{k}, \mathrm{p}},(113.3,113.1,112.5,112.1)_{\mathrm{e}, \mathrm{h}, \mathrm{l}, \mathrm{o}}, 105.5_{\mathrm{b}}$ $(68.9,68.7,68.4,68.3)_{\mathrm{a}, \mathrm{q}},(31.9,31.8,31.3,31.2,30.8,30.2,29.7,29.6,29.5,29.4,29.3,29.2,29.0$, $28.9,28.8,28.7,28.6,28.5,26.1,26.0,25.5,25.4,22.6,22.0)_{\mathrm{r}, \mathrm{s}},(14.0,13.4)_{\mathrm{t}} \mathrm{ppm} . \mathrm{MS}(\mathrm{FAB}+) \mathrm{m} / z$ : $1838\left[\mathrm{M}^{+\cdot}\right]$.

\section{Dichloro[bis(3-(3',4',5'-tridecyloxyphenyl)-5-(3',,4',-didecyloxyphenyl)pyrazole-1-}

yl)methane Jzinc(II) 22: Dissolved in hexane. Yield: > 90\%. Anal. Calcd for $\mathrm{C}_{131} \mathrm{H}_{224} \mathrm{Cl}_{2} \mathrm{~N}_{4} \mathrm{O}_{10} \mathrm{Zn}$ : C 73.13, H 10.49, N 2.60. Found: C 73.04, H 10.43, 2.60. IR (Thin film): 1583, 1497 (arC-C), 1260, 1241 $(\mathrm{C}-\mathrm{O}) \mathrm{cm}^{-1} .{ }^{1} \mathrm{H}$ NMR $\left(400 \mathrm{MHz}, \mathrm{CDCl}_{3}\right) \delta 7.44\left(\mathrm{~d}, J=2.4 \mathrm{~Hz}, 1 \mathrm{Ht}_{\mathrm{l}}{ }^{\prime}\right), 7.27(\mathrm{dd}, J=8.8 \mathrm{~Hz}, J=2.0 \mathrm{~Hz}$, $\left.1 \mathrm{Ht}_{\mathrm{p}}\right), 7.01\left(\mathrm{~s}, 2 \mathrm{Hc}_{\mathrm{e}, \mathrm{i}}\right), 7.00\left(\mathrm{~s}, 2 \mathrm{Ht}_{\mathrm{e}, \mathrm{i}}\right), 6.91\left(\mathrm{~d}, J=8.8 \mathrm{~Hz}, 1 \mathrm{Ht}_{\mathrm{o}^{\prime}}\right), 6.76\left(\mathrm{~d}, J=2.0 \mathrm{~Hz}, 1 \mathrm{Hc}_{1}\right), 6.70(\mathrm{~d}, J=$ 
$\left.2.0 \mathrm{~Hz}, 1 \mathrm{Ht}_{\mathrm{l}}\right), 6.56\left(\mathrm{~d}, J=8.4 \mathrm{~Hz}, 1 \mathrm{Hc}_{\mathrm{o}}\right), 6.53\left(\mathrm{~s}, 2 \mathrm{Ht}_{\mathrm{a}}\right), 6.50\left(\mathrm{~s}, 3 \mathrm{Hc}_{\mathrm{a}, \mathrm{b}, \mathrm{b}}, \mathrm{t}_{\mathrm{b}}\right), 6.47\left(\mathrm{~s}, 1 \mathrm{Ht}_{\mathrm{b}}\right), 6.43(\mathrm{dd}, J=$ $\left.8.0 \mathrm{~Hz}, J=2.0 \mathrm{~Hz}, 1 \mathrm{Hc}_{\mathrm{p}}\right), 6.42\left(\mathrm{~s}, 2 \mathrm{Ht}_{\mathrm{e}^{\prime}, \mathrm{i}^{\prime}}\right), 6.37\left(\mathrm{~d}, J=8.4 \mathrm{~Hz}, 1 \mathrm{Ht}_{\mathrm{o}}\right), 6.13(\mathrm{dd}, J=8.0 \mathrm{~Hz}, J=2.0 \mathrm{~Hz}$, $\left.1 \mathrm{Ht}_{\mathrm{p}}\right), 4.19\left(\mathrm{t}, J=6.4 \mathrm{~Hz}, 2 \mathrm{Hc}_{\mathrm{q}}\right), 4.11\left(\mathrm{t}, J=6.4 \mathrm{~Hz}, 4 \mathrm{Ht}_{\mathrm{q}}\right), 4.10\left(\mathrm{t}, J=6.4 \mathrm{~Hz}, 4 \mathrm{Ht}_{\mathrm{q}}\right), 3.85-4.03(\mathrm{~m}$, $\left.20 \mathrm{H}_{\mathrm{q}}\right), 1.70-1.89\left(\mathrm{~m}, 30 \mathrm{H}_{\mathrm{r}}\right), 1.27-1.48\left(\mathrm{~m}, 210 \mathrm{H}_{\mathrm{s}}\right), 0.87\left(\mathrm{~m}, 45 \mathrm{H}_{\mathrm{t}}\right) \mathrm{ppm} .{ }^{13} \mathrm{C} \mathrm{NMR}\left(100 \mathrm{MHz}, \mathrm{CDCl}_{3}\right) \delta$ $(156.7,156.5)_{\mathrm{c}, \mathrm{j}},(153.9,153.3)_{\mathrm{f}, \mathrm{h}},(150.9,149.6,149.2,148.1,147.5,147.0)_{\mathrm{m}, \mathrm{n}, \mathrm{c}, \mathrm{j}},(139.95,139.9$, $139.8)_{\mathrm{g}},(124.3,124.2,122.1,121.3,120.8,119.1,118.1,115.9,114.0,112.1)_{\mathrm{d}, \mathrm{k}, \mathrm{l}, \mathrm{o}, \mathrm{p}},(107.1,106.9$, 106.6) $)_{\mathrm{e}, \mathrm{i}}, 105.9_{\mathrm{b}},(73.5,69.7,69.5,69.4,69.0,68.9)_{\mathrm{q}}, 57.5_{\mathrm{a}},(31.9,30.6,30.4,29.9,29.8,29.78,29.7$, 29.66, 29.5, 29.47, 29.4, 29.3, 26.2, 26.1, 26.05, 22.7) $)_{\mathrm{r}, \mathrm{s}}, 14.1_{\mathrm{t}} \mathrm{ppm} . \mathrm{MS}(\mathrm{FAB}+) \mathrm{m} / z: 2116$ [M-Cl].

Dichloro[bis(3,5-bis(3',4',5'-tridecyloxyphenyl)pyrazole-1-yl)methane]zinc(II) 23: Dissolved in hexane. Yield: > 90\%. Anal. Calcd for $\mathrm{C}_{151} \mathrm{H}_{264} \mathrm{Cl}_{2} \mathrm{~N}_{4} \mathrm{O}_{12} \mathrm{Zn}$ : C 73.60, H 10.80, N 2.27. Found: C 73.23, $\mathrm{H}$ 10.64, 2.19. IR (nujol, NaCl): 1582, 1492 (arC-C), $1240(\mathrm{C}-\mathrm{O}) \mathrm{cm}^{-1} .{ }^{1} \mathrm{H}$ NMR $\left(400 \mathrm{MHz}, \mathrm{CDCl}_{3}\right) \delta$ $6.9\left(\mathrm{~s}, 4 \mathrm{H}_{\mathrm{e}, \mathrm{i}}\right), 6.7\left(\mathrm{~s}, 2 \mathrm{H}_{\mathrm{a}}\right), 6.4\left(\mathrm{~s}, 2 \mathrm{H}_{\mathrm{b}}\right), 6.2\left(\mathrm{~s}, 4 \mathrm{H}_{\mathrm{l}, \mathrm{p}}\right), 4.1\left(\mathrm{t}, J=6.4 \mathrm{~Hz}, 8 \mathrm{H}_{\mathrm{q}}\right), 3.98\left(\mathrm{t}, J=6.0 \mathrm{~Hz}, 4 \mathrm{H}_{\mathrm{q}}\right)$, $3.98\left(\mathrm{t}, J=6.4 \mathrm{~Hz}, 4 \mathrm{H}_{\mathrm{q}}\right), 3.78\left(\mathrm{t}, J=6.4 \mathrm{~Hz}, 8 \mathrm{H}_{\mathrm{q}}\right), 1.66-1.82\left(\mathrm{~m}, 24 \mathrm{H}_{\mathrm{r}}\right), 1.26-1.47\left(\mathrm{~m}, 168 \mathrm{H}_{\mathrm{s}}\right), 0.88$ $\left(\mathrm{m}, 36 \mathrm{H}_{\mathrm{t}}\right)$ ppm. ${ }^{13} \mathrm{C} \mathrm{NMR}\left(100 \mathrm{MHz}, \mathrm{CDCl}_{3}\right) \delta 156.0_{\mathrm{j}},(153.6,153.3)_{\mathrm{f}, \mathrm{h}, \mathrm{m}, \mathrm{o}}, 149.2_{\mathrm{c}}, 139.9_{\mathrm{g}, \mathrm{n}},(124.2$, $121.2)_{\mathrm{d}, \mathrm{k}},(109.7,107.1)_{\mathrm{e}, \mathrm{i}, \mathrm{l}, \mathrm{p}}, 106.5_{\mathrm{b}},(73.5,72.1,69.4,69.4,68.9)_{\mathrm{q}}, 57.6_{\mathrm{a}},(32.2,32.1,31.4,29.7,29.5$, 29.4, 29.0, 28.9, 28.8, 26.1, 22.7) $)_{\mathrm{r}, \mathrm{s}}, 14.1_{\mathrm{t}} \mathrm{ppm} . \mathrm{MS}(\mathrm{MALDI}+) \mathrm{m} / z: 2428$ [M-Cl].

Dichloro[bis(3,5-bis(3',4',5'-trimethoxyphenyl)pyrazole-1-yl)methane]zinc(II) 24: Dissolved in hexane. Yield: > 90\%. Anal. Calcd for $\mathrm{C}_{43} \mathrm{H}_{48} \mathrm{Cl}_{2} \mathrm{~N}_{4} \mathrm{O}_{12} \mathrm{Zn}$ : C 54.41, H 5.10, N 5.90. Found: C 54.05, $\mathrm{H}$ 4.91, N 5.75. IR (nujol, $\mathrm{NaCl}): 1613,1498$ (arC-C), $1251(\mathrm{C}-\mathrm{O}) \mathrm{cm}^{-1} .{ }^{1} \mathrm{H}$ NMR $\left(300 \mathrm{MHz}, \mathrm{CDCl}_{3}\right) \delta$ $7.00\left(\mathrm{~s}, 4 \mathrm{H}_{\mathrm{e}}\right), 6.74\left(\mathrm{~s}, 2 \mathrm{H}_{\mathrm{a}}\right), 6.49\left(\mathrm{~s}, 2 \mathrm{H}_{\mathrm{b}}\right), 6.32\left(\mathrm{~s}, 4 \mathrm{H}_{\mathrm{l}}\right), 3.95\left(\mathrm{~s}, 6 \mathrm{H}_{\mathrm{q}}\right), 3.90\left(\mathrm{~s}, 12 \mathrm{H}_{\mathrm{q}}\right), 3.88\left(\mathrm{~s}, 12 \mathrm{H}_{\mathrm{q}}\right)$, $3.79\left(\mathrm{~s}, 6 \mathrm{H}_{\mathrm{q}}\right) \mathrm{ppm} .{ }^{13} \mathrm{C} \mathrm{NMR}\left(100 \mathrm{MHz}, \mathrm{CDCl}_{3}\right) \delta(153.6,153.4)_{\mathrm{f}, \mathrm{i}, \mathrm{m}}, 147.1_{3}, 139.7_{\mathrm{g}, \mathrm{n}}(\mathrm{b}),(124.7$, $121.6)_{\mathrm{d}, \mathrm{k}},(106.7,106.3)_{\mathrm{e}, \mathrm{l}}, 105.6_{\mathrm{b}}, 60.9_{\mathrm{q}}, 56.4_{\mathrm{q}}, 56.3_{\mathrm{a}} \mathrm{ppm}$. MS (MALDI+) m/z: 911 [M-Cl]. 
NOE spectra for $\mathbf{1 2}$.

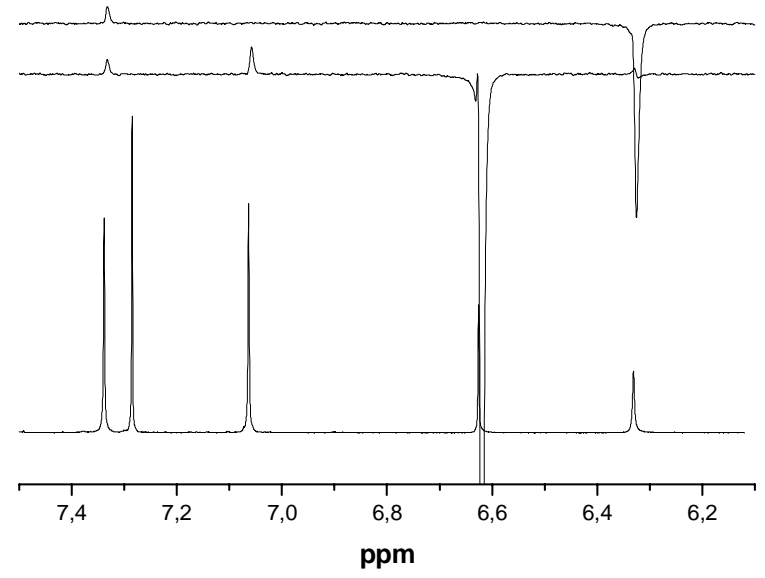


${ }^{1} \mathrm{H}$ NMR spectra for the characterization of isomers in $\mathbf{8}$ and $\mathbf{2 0 .}$
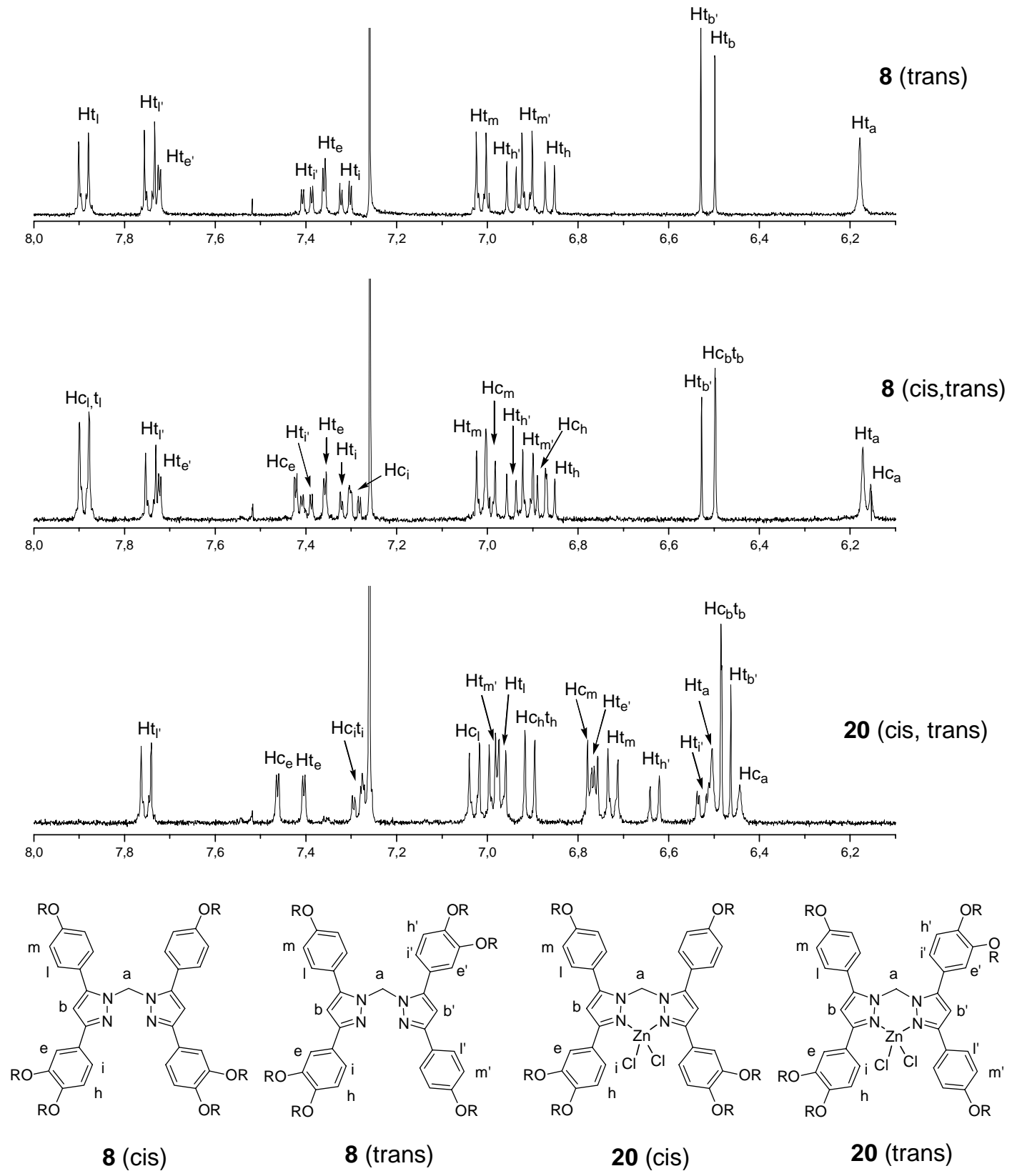
Variable temperature ${ }^{1} \mathrm{H}$ NMR spectra for $\mathbf{1 8}$.

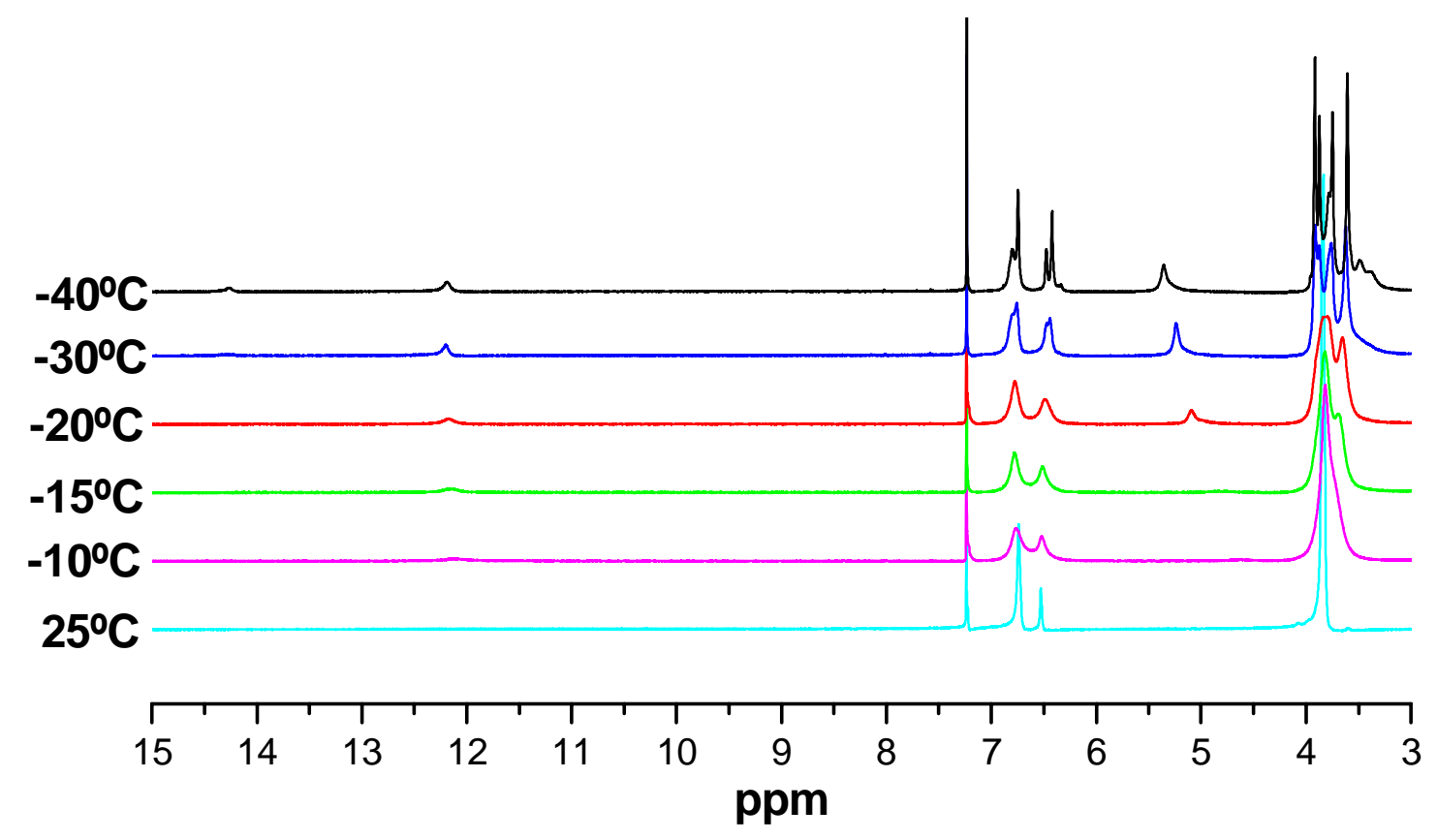


ORTEP molecular drawing with atom numbering scheme for compound $\mathbf{1 8 .}$

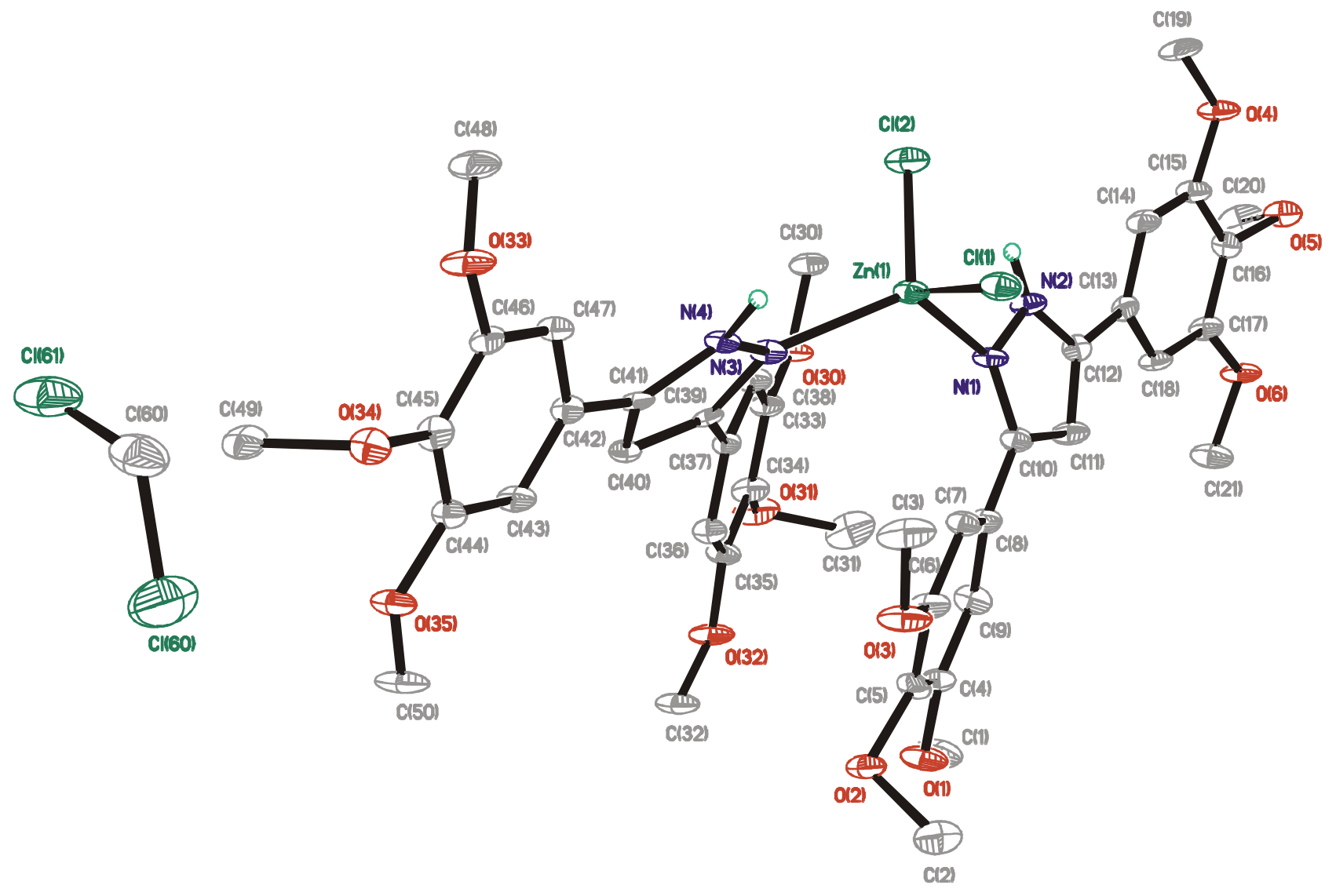


Representative bond distances and angles for compound 18.

\begin{tabular}{llll}
\multicolumn{2}{l}{ Distances $[\AA ̊]$} \\
\hline $\mathrm{Zn}(1)-\mathrm{N}(1)$ & $1.972(2)$ & $\mathrm{Zn}(1)-\mathrm{Cl}(2)$ & $2.1568(9)$ \\
$\mathrm{Zn}(1)-\mathrm{N}(3)$ & $2.177(3)$ & $\mathrm{Zn}(1)-\mathrm{Cl}(1)$ & $2.2848(11)$ \\
$\mathrm{N}(1)-\mathrm{C}(10)$ & $1.242(3)$ & $\mathrm{N}(1)-\mathrm{N}(2)$ & $1.385(3)$ \\
$\mathrm{N}(2)-\mathrm{C}(12)$ & $1.300(3)$ & $\mathrm{N}(3)-\mathrm{C}(39)$ & $1.348(4)$ \\
$\mathrm{N}(3)-\mathrm{N}(4)$ & $1.349(3)$ & $\mathrm{N}(4)-\mathrm{C}(41)$ & $1.409(4)$ \\
$\mathrm{C}(10)-\mathrm{C}(11)$ & $1.413(4)$ & $\mathrm{C}(11)-\mathrm{C}(12)$ & $1.316(4)$ \\
$\mathrm{C}(39)-\mathrm{C}(40)$ & $1.468(4)$ & $\mathrm{C}(40)-\mathrm{C}(41)$ & $1.318(4)$
\end{tabular}

Angles [ำ]

\begin{tabular}{llll}
\hline \hline $\mathrm{N}(1)-\mathrm{Zn}(1)-\mathrm{Cl}(2)$ & $93.25(7)$ & $\mathrm{N}(1)-\mathrm{Zn}(1)-\mathrm{N}(3)$ & $119.45(10)$ \\
$\mathrm{Cl}(2)-\mathrm{Zn}(1)-\mathrm{N}(3)$ & $114.50(8)$ & $\mathrm{N}(1)-\mathrm{Zn}(1)-\mathrm{Cl}(1)$ & $107.49(10)$ \\
$\mathrm{Cl}(2)-\mathrm{Zn}(1)-\mathrm{Cl}(1)$ & $115.75(4)$ & $\mathrm{N}(3)-\mathrm{Zn}(1)-\mathrm{Cl}(1)$ & $106.34(8)$ \\
$\mathrm{C}(10)-\mathrm{N}(1)-\mathrm{N}(2)$ & $103.8(2)$ & $\mathrm{C}(10)-\mathrm{N}(1)-\mathrm{Zn}(1)$ & $137.5(2)$ \\
$\mathrm{N}(2)-\mathrm{N}(1)-\mathrm{Zn}(1)$ & $118.65(17)$ & $\mathrm{C}(12)-\mathrm{N}(2)-\mathrm{N}(1)$ & $117.0(2)$ \\
$\mathrm{C}(39)-\mathrm{N}(3)-\mathrm{N}(4)$ & $98.6(2)$ & $\mathrm{C}(39)-\mathrm{N}(3)-\mathrm{Zn}(1)$ & $137.42(18)$ \\
$\mathrm{N}(4)-\mathrm{N}(3)-\mathrm{Zn}(1)$ & $122.6(2)$ & $\mathrm{N}(3)-\mathrm{N}(4)-\mathrm{C}(41)$ & $116.3(3)$ \\
$\mathrm{N}(1)-\mathrm{C}(10)-\mathrm{C}(11)$ & $107.3(3)$ & $\mathrm{N}(2)-\mathrm{C}(12)-\mathrm{C}(11)$ & $99.1(2)$ \\
$\mathrm{N}(3)-\mathrm{C}(39)-\mathrm{C}(40)$ & $115.7(3)$ & $\mathrm{C}(41)-\mathrm{C}(40)-\mathrm{C}(39)$ & $102.7(3)$ \\
$\mathrm{C}(40)-\mathrm{C}(41)-\mathrm{N}(4)$ & $106.6(3)$ & & \\
& & &
\end{tabular}


ORTEP molecular drawing with atom numbering scheme for compound 24.

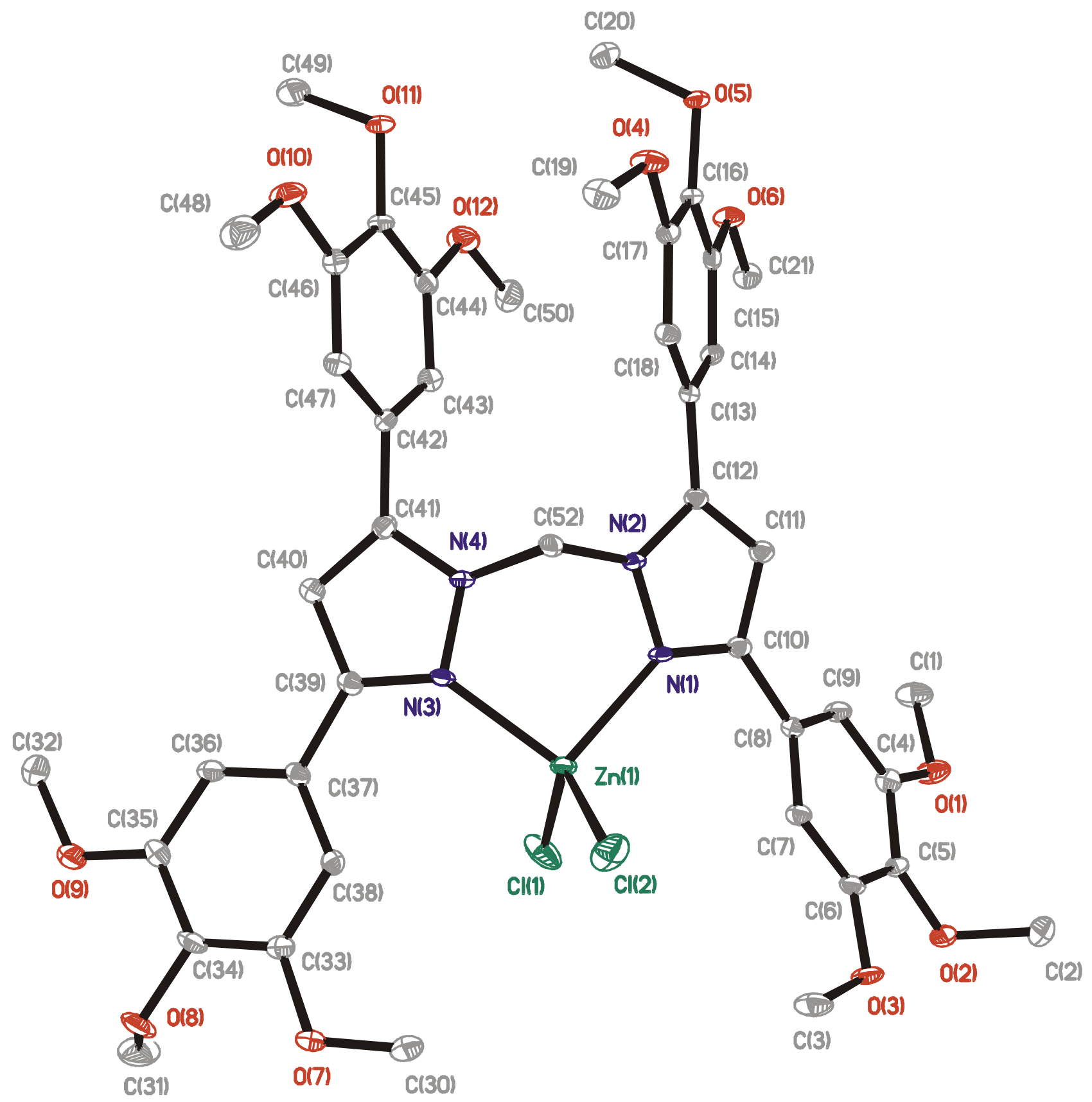


Representative bond distances and angles for compound $\mathbf{2 4}$.

\begin{tabular}{|c|c|c|c|c|c|}
\hline \multicolumn{6}{|c|}{ Distances $[\AA ̊]$} \\
\hline $\mathrm{Zn}(1)-\mathrm{N}(3)$ & \multicolumn{2}{|c|}{$2.0804(17)$} & $\mathrm{Zn}(1)-\mathrm{N}(1)$ & \multicolumn{2}{|c|}{$2.0823(14)$} \\
\hline $\mathrm{Zn}(1)-\mathrm{Cl}(1)$ & \multicolumn{2}{|c|}{$2.2000(6)$} & $\mathrm{Zn}(1)-\mathrm{Cl}(2)$ & \multicolumn{2}{|c|}{$2.2131(6)$} \\
\hline$N(1)-C(10)$ & \multicolumn{2}{|c|}{$1.343(3)$} & $N(1)-N(2)$ & \multicolumn{2}{|c|}{$1.372(2)$} \\
\hline$N(2)-C(12)$ & \multicolumn{2}{|c|}{$1.364(2)$} & $N(2)-C(52)$ & \multicolumn{2}{|c|}{$1.448(3)$} \\
\hline$N(3)-C(39)$ & \multicolumn{2}{|c|}{$1.338(3)$} & $N(3)-N(4)$ & \multicolumn{2}{|c|}{$1.3775(19)$} \\
\hline$N(4)-C(41)$ & \multicolumn{2}{|c|}{$1.344(3)$} & $N(4)-C(52)$ & \multicolumn{2}{|c|}{$1.444(2)$} \\
\hline$C(10)-C(11)$ & \multicolumn{2}{|c|}{$1.402(2)$} & $C(11)-C(12)$ & \multicolumn{2}{|c|}{$1.364(3)$} \\
\hline$C(39)-C(40)$ & \multicolumn{2}{|c|}{$1.406(3)$} & $C(40)-C(41)$ & \multicolumn{2}{|c|}{$1.370(2)$} \\
\hline \multicolumn{6}{|l|}{ Angles [ํ] } \\
\hline \multicolumn{2}{|c|}{$\mathrm{N}(3)-\mathrm{Zn}(1)-\mathrm{N}(1)$} & $95.65(6)$ & \multicolumn{2}{|c|}{$\mathrm{N}(3)-\mathrm{Zn}(1)-\mathrm{Cl}(1)$} & $108.25(5)$ \\
\hline \multicolumn{2}{|c|}{$\mathrm{N}(1)-\mathrm{Zn}(1)-\mathrm{Cl}(1)$} & $114.29(5)$ & \multicolumn{2}{|c|}{$\mathrm{N}(3)-\mathrm{Zn}(1)-\mathrm{Cl}(2)$} & $115.52(5)$ \\
\hline \multicolumn{2}{|c|}{$\mathrm{N}(1)-\mathrm{Zn}(1)-\mathrm{Cl}(2)$} & $107.34(5)$ & \multicolumn{2}{|c|}{$\mathrm{Cl}(1)-\mathrm{Zn}(1)-\mathrm{Cl}(2)$} & $114.45(3)$ \\
\hline \multicolumn{2}{|c|}{$C(10)-N(1)-N(2)$} & $105.99(14)$ & \multicolumn{2}{|c|}{$C(10)-N(1)-Z n(1)$} & $132.97(12)$ \\
\hline \multicolumn{2}{|c|}{$N(2)-N(1)-Z n(1)$} & $119.00(12)$ & \multicolumn{2}{|c|}{$N(4)-N(3)-Z n(1)$} & $115.03(12)$ \\
\hline \multicolumn{2}{|c|}{$\mathrm{C}(41)-\mathrm{N}(4)-\mathrm{N}(3)$} & $111.75(15)$ & \multicolumn{2}{|c|}{$\mathrm{C}(41)-\mathrm{N}(4)-\mathrm{C}(52)$} & $127.49(15)$ \\
\hline \multicolumn{2}{|c|}{$N(3)-N(4)-C(52)$} & $119.05(16)$ & \multicolumn{2}{|c|}{$N(1)-C(10)-C(11)$} & $109.50(17)$ \\
\hline \multicolumn{2}{|c|}{$C(11)-C(12)-N(2)$} & $106.69(15)$ & \multicolumn{2}{|c|}{$C(12)-C(11)-C(10)$} & $107.07(17)$ \\
\hline \multicolumn{2}{|c|}{$\mathrm{C}(41)-\mathrm{C}(40)-\mathrm{C}(39)$} & $106.40(18)$ & \multicolumn{2}{|c|}{$N(4)-C(41)-C(40)$} & $106.73(16)$ \\
\hline$N(4)-C(52)-\wedge$ & (2) & $112.36(16)$ & & & \\
\hline
\end{tabular}


